\title{
Generation of a Triple-Transgenic Zebrafish Line for Assessment of Developmental Neurotoxicity during Neuronal Differentiation
}

\author{
Junko Koiwa ${ }^{1}$, Takashi Shiromizu ${ }^{1}$, Yuka Adachi ${ }^{1}$, Makoto Ikejiri ${ }^{2}$, Kaname Nakatani ${ }^{3}$, \\ Toshio Tanaka ${ }^{4}$ and Yuhei Nishimura ${ }^{1, *(\mathbb{D})}$ \\ 1 Department of Integrative Pharmacology, Mie University Graduate School of Medicine, Tsu, Mie 514-8507, \\ Japan; j-koiwa@doc.medic.mie-u.ac.jp (J.K.); tshiromizu@doc.medic.mie-u.ac.jp (T.S.); \\ 319m001@m.mie-u.ac.jp (Y.A.) \\ 2 Department of Central Laboratory, Mie University Hospital, Tsu, Mie 514-8507, Japan; \\ mktik@clin.medic.mie-u.ac.jp \\ 3 Department of Genomic Medicine, Mie University Hospital, Tsu, Mie 514-8507, Japan; \\ nakatani@clin.medic.mie-u.ac.jp \\ 4 Department of Systems Pharmacology, Mie University Graduate School of Medicine, Tsu, Mie 514-8507, \\ Japan; tanaka@doc.medic.mie-u.ac.jp \\ * Correspondence: yuhei@doc.medic.mie-u.ac.jp
}

Received: 1 August 2019; Accepted: 22 September 2019; Published: 24 September 2019

\begin{abstract}
The developing brain is extremely sensitive to many chemicals. Exposure to neurotoxicants during development has been implicated in various neuropsychiatric and neurological disorders, including autism spectrum disorders and schizophrenia. Various screening methods have been used to assess the developmental neurotoxicity (DNT) of chemicals, with most assays focusing on cell viability, apoptosis, proliferation, migration, neuronal differentiation, and neuronal network formation. However, assessment of toxicity during progenitor cell differentiation into neurons, astrocytes, and oligodendrocytes often requires immunohistochemistry, which is a reliable but labor-intensive and time-consuming assay. Here, we report the development of a triple-transgenic zebrafish line that expresses distinct fluorescent proteins in neurons (Cerulean), astrocytes (mCherry), and oligodendrocytes (mCitrine), which can be used to detect DNT during neuronal differentiation. Using in vivo fluorescence microscopy, we could detect DNT by 6 of the 10 neurotoxicants tested after exposure to zebrafish from $12 \mathrm{~h}$ to 5 days' post-fertilization. Moreover, the chemicals could be clustered into three main DNT groups based on the fluorescence pattern: (i) inhibition of neuron and oligodendrocyte differentiation and stimulation of astrocyte differentiation; (ii) inhibition of neuron and oligodendrocyte differentiation; and (iii) inhibition of neuron and astrocyte differentiation, which suggests that reporter expression reflects the toxicodynamics of the chemicals. Thus, the triple-transgenic zebrafish line developed here may be a useful tool to assess DNT during neuronal differentiation.
\end{abstract}

Keywords: developmental neurotoxicity; neuronal differentiation; zebrafish; in vivo fluorescence imaging

\section{Introduction}

The developing brain is more vulnerable than the adult brain to most chemicals [1-6]. Exposure to neurotoxicants during development has been implicated in various neurodevelopmental and neuropsychiatric diseases, including autism spectrum disorders, attention deficit hyperactive disorder, learning disabilities, and schizophrenia [7-15]. The Organisation for Economic Co-operation and Development (OECD) has developed guidelines to assess the developmental neurotoxicity (DNT) of 
chemicals, with rodents as the model animal of choice [16]. However, while rodents have contributed significantly to our understanding of DNT [3,17-19], experiments using large numbers of rodents are time-consuming, expensive, and accompanied by ethical concerns. Thus, alternative test systems, such as human stem/progenitor cell and zebrafish models, have the potential to enable testing of large chemical libraries while reducing expense and minimizing rodent use [4,20-25].

In human stem/progenitor cell models, various endpoints have been used to assess DNT, including viability, apoptosis, proliferation, migration, differentiation, and neuronal network formation [24,26,27]. These endpoints facilitate the identification of adverse outcome pathways and the development of integrated approaches to test and assess chemical-induced DNT [22,28]. Differentiation of neural stem/progenitor cells into neurons, astrocytes, and oligodendrocytes has been analyzed by immunohistochemical staining with antibodies against cell-specific proteins such as NeuN/RNA-binding Fox3 (Rbfox3) for neurons, glial fibrillary acidic protein (GFAP) for astrocytes, and myelin basic protein (MBP) for oligodendrocytes [26,29]. Reporter assays in which expression of fluorescent proteins is driven by the promoters of genes selectively expressed in neurons, astrocytes, and oligodendrocytes have also been used to assess the effects of developmental neurotoxicants on cell differentiation [30]. Fluorescent reporter assays do not require time-consuming procedures such as fixation and immunostaining, and they can be performed in a relatively high-throughput manner. However, distinguishing between multiple cell types requires a multiplexed fluorescent reporter system and, although this has been employed in some human stem/progenitor cell models [31], to our knowledge, it has not been used to distinguish between neurons, astrocytes, and oligodendrocytes in the same organism.

Compared with other species, zebrafish offer several advantages as a model system for DNT testing. For example, the pattern of developmental gene expression and structure of various brain regions is relatively conserved in zebrafish; a wide range of chemicals can be absorbed from the surrounding medium; the small size and prolific breeding capacity lowers housing and experimental costs [4,23,32-35]; and the optical transparency, particularly of pigmentless mutants, make zebrafish suitable for in vivo fluorescence imaging $[4,20]$. To date, many transgenic $(\mathrm{Tg})$ zebrafish lines have been developed that express fluorescent proteins in specific neuronal subtypes [36-38], and multiplexed reporter systems have also been implemented in this species [39]. To facilitate DNT testing, we have developed a triple-fluorescent $\mathrm{Tg}$ zebrafish line in which progenitor cell differentiation into neurons, astrocytes, and oligodendrocytes is accompanied by cell type-specific expression of the fluorescent proteins Cerulean, mCherry, and mCitrine, respectively, thereby enabling the effects of neurotoxicants on neuronal differentiation to be assessed in vivo.

\section{Results}

2.1. Construction of Transposon Vectors to Express Cerulean, Mcherry, and Mcitrine in Neurons, Astrocytes, and Oligodendrocytes

We selected the Tol2 system to selectively express the three fluorescent proteins in neurons, astrocytes, and oligodendrocytes [40]. Tol2 is an autonomous transposon that can catalyze transposition of a DNA construct flanked by Tol2 sequences into the host genome. Cerulean, mCherry, and mCitrine were selected as the reporter proteins because their fluorescent signals can be distinguished using CFP, RFP, and YFP filters. To direct cell type-specific reporter expression, we used the regulatory sequences of enolase 2 (eno2), gfap, and $\mathrm{mbp}$, which are selectively expressed in neurons, astrocytes, and oligodendrocytes, respectively [36-38]. The regulatory sequence of eno2 gfap, or mbp was cloned upstream of the coding region of Cerulean, mCherry, or mCitrine, respectively, and then inserted between two Tol2 sequences in the vector backbone, generating eno2: Cerulean, gfap:mCherry, and mbp:mCitrine vectors (Figure 1). 
eno2: Cerulean

$1 \mathrm{~kb}$

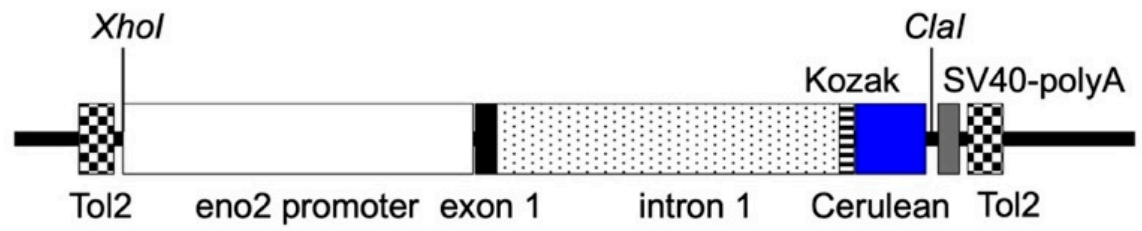

\section{gfap: mCherry}

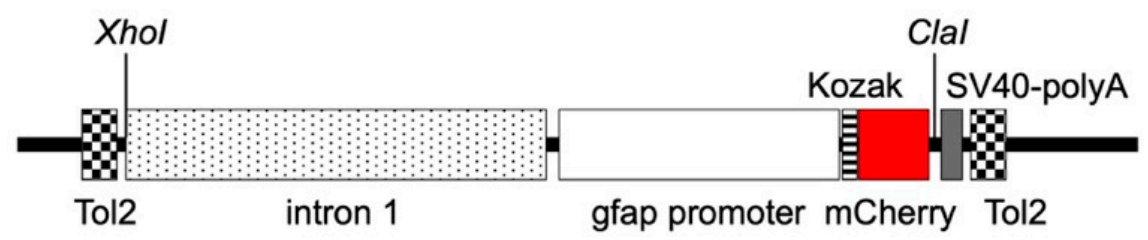

\section{mbp: mCitrine}

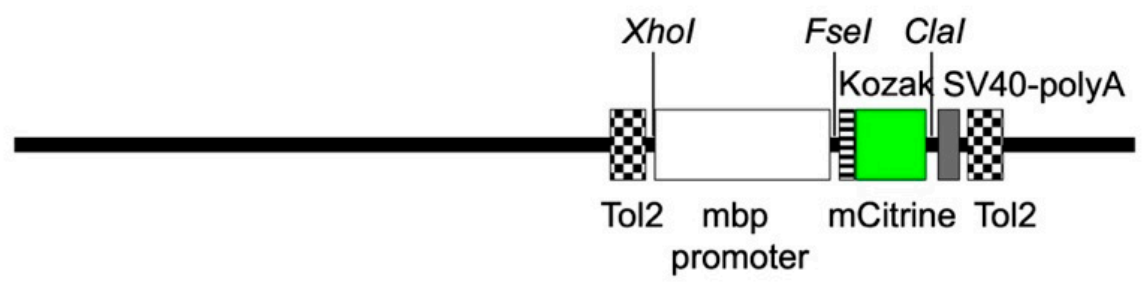

Figure 1. Schematic representation of transposon vectors.

The coding regions of Cerulean, mCherry, and mCitrine were placed downstream of the regulatory sequences of eno2, gfap, and mbp, and cloned between two Tol2 sequences in the vector backbone to direct selective protein expression in neurons, astrocytes, and oligodendrocytes, respectively. The resulting Tg (eno2:Cerulean, gfap:mCherry, mbp:mCitrine) zebrafish line is referred to as the triple-Tg line.

\subsection{Generation of Triple-Tg Zebrafish}

One of the three transposon vectors was injected together with transposase mRNA into fertilized zebrafish eggs to generate three lines expressing a single fluorescent protein (Cerulean, mCherry, or mCitrine) in the central nervous system (CNS). At maturity, the single-Tg zebrafish were mated to generate double- $\mathrm{Tg}$ zebrafish. In turn, the adult double- $\mathrm{Tg}$ zebrafish were mated to generate triple- $\mathrm{Tg}$ zebrafish expressing Cerulean, mCherry, and mCitrine in the CNS. Representative in vivo images of the larvae at 5 days' post-fertilization (dpf) is shown in Figure 2. The fluorescence patterns obtained are consistent with the results of previous studies of single fluorescent protein expression in zebrafish neurons, astrocytes, or oligodendrocytes driven by eno2, gfap, or mbp promoters, respectively [36-38]. These results suggest that the fluorescent proteins are selectively expressed in neurons, astrocytes, and oligodendrocytes. 


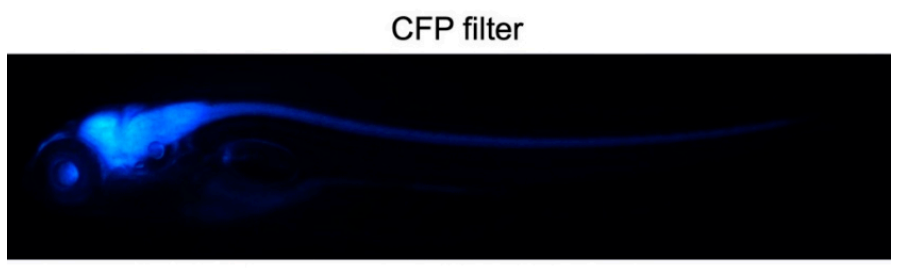

RFP filter

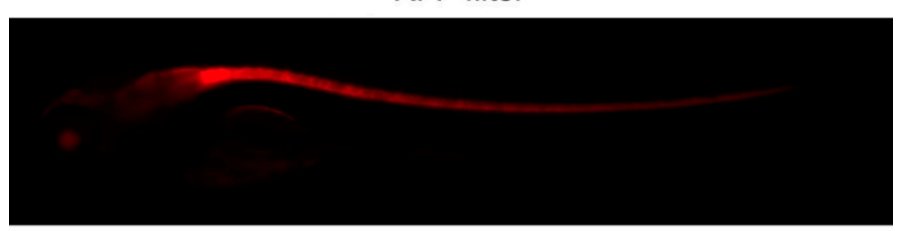

YFP filter
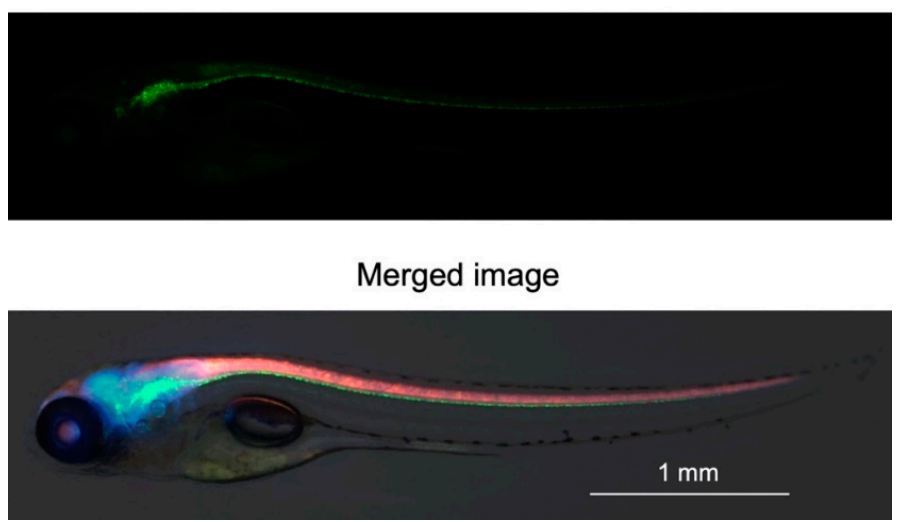

Figure 2. In vivo fluorescence imaging of the triple-Tg zebrafish line.

Representative in vivo fluorescence images of triple-Tg zebrafish at $5 \mathrm{dpf}$.

\subsection{Assessment of DNT Using Triple-Tg Zebrafish}

To assess the DNT of a panel of chemicals, we first measured the no observed effect concentration (NOEC) for lethality, which is the highest concentration of each chemical to which zebrafish are exposed that does not significantly affect the lethality compared with the controls. Triple-Tg zebrafish were exposed to $\log$ serial dilutions of 13 chemicals from $12 \mathrm{~h}$ post-fertilization (hpf) to $5 \mathrm{dpf}$ and then imaged at $5 \mathrm{dpf}$. The effects of the chemicals on lethality were evaluated according to a Fish Embryo Acute Toxicity Test guideline (OECD TG236) [41]. The measured NOEC for lethality of each chemical was designated the maximum tolerable concentration (MTC) and was used for DNT assessment. We employed 13 chemicals considered suitable for DNT test method validation [42,43], of which 10 were established developmental neurotoxicants: valproic acid (VPA), trichostatin A (TSA), carbamazepine (CBZ), nicotine (NCT), chlorpyrifos (CPF), cyclopamine (CPM), methyl mercury (MeHg), dexamethasone (DEX), retinoic acid (RA), and bisphenol A (BPA); and 3 were not neurotoxicants: deferoxamine (DFX), saccharin (SAC), and acetaminophen (APAP). We selected the 10 developmental neurotoxicants because they have been reported to affect neuronal differentiation [44-56]. The MTCs for these chemicals were $1 \mathrm{nM}$ for RA, $100 \mathrm{nM}$ for TSA, CPM, and MeHg, $10 \mu \mathrm{M}$ for CPF, $100 \mu \mathrm{M}$ for VPA, CBZ, DEX, and SAC, and $1000 \mu \mathrm{M}$ for DFX and APAP (Figure 3). 


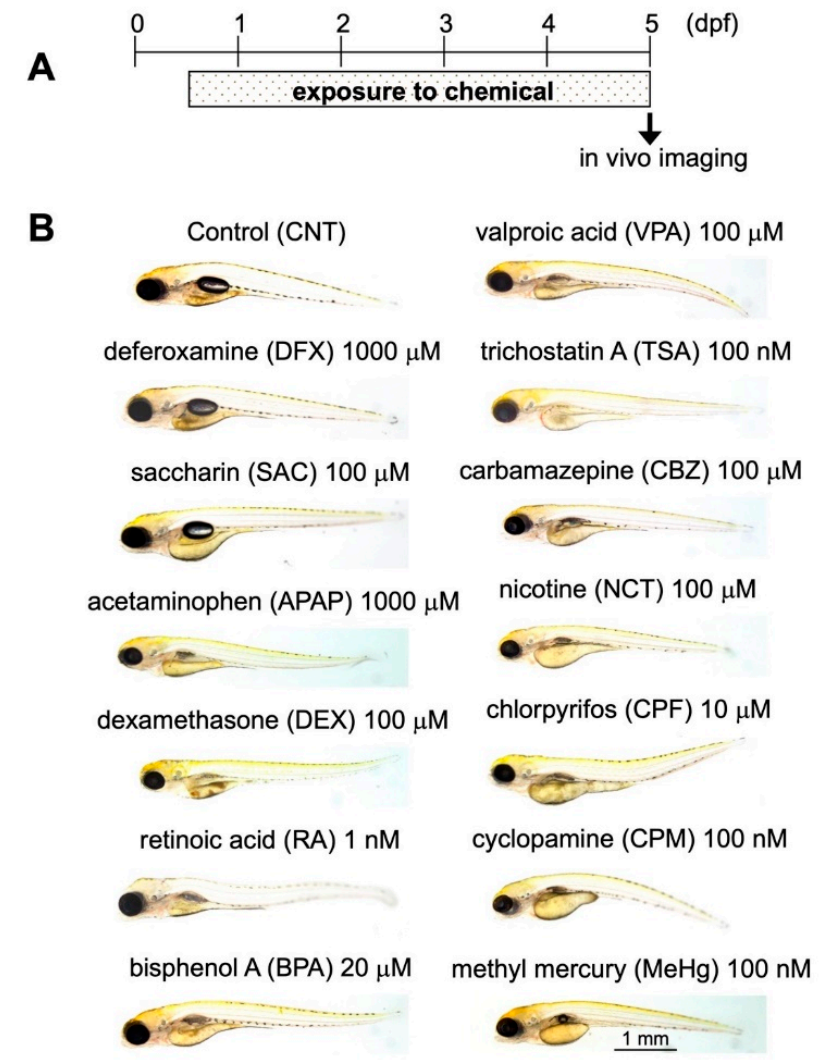

Figure 3. Bright-field images of triple-Tg zebrafish exposed to the maximum tolerable concentrations of chemicals during early development. (A) Experimental protocol. (B) Triple-Tg zebrafish were treated with the indicated chemicals at their maximum tolerable concentrations from $12 \mathrm{hpf}$ to $5 \mathrm{dpf}$. The animals were then anesthetized and subjected to in vivo bright-field imaging using a stereomicroscope.

We next performed in vivo fluorescence imaging of the triple-Tg zebrafish after exposure to the 13 chemicals at their MTCs from $12 \mathrm{hpf}$ to $5 \mathrm{dpf}$. The fluorescent signals were then quantified and normalized to the values for untreated control zebrafish (Figure 4 and Supplemental Table S1). We found that Cerulean fluorescence (CFP) was significantly reduced by exposure of zebrafish to TSA, CBZ, NCT, BPA, and CPF (Figure 4A and Supplemental Table S1), whereas mCherry fluorescence (RFP) was significantly increased by VPA and TSA and significantly decreased by BPA (Figure 4B and Supplemental Table S1), and mCitrine fluorescence (YFP) was significantly decreased by VPA, TSA, CBZ, and NCT (Figure 4C and Supplemental Table S1). To translate the fluorescent protein expression patterns into functional effects on neuronal differentiation, we calculated the ratios of the fluorescent signals. VPA, TSA, and CBZ significantly increased the RFP/CFP ratio and decreased the YFP/CFP and YFP/RFP ratios (Figure 4D-F and Supplemental Table S1), suggesting that these compounds increased and decreased the differentiation of progenitors into astrocytes and oligodendrocytes, respectively. NCT significantly decreased the ratios of YFP/CFP and YFP/RFP, but not RFP/CFP (Figure 4D-F and Supplemental Table S1), suggesting that NCT preferentially interfered with the differentiation into oligodendrocytes. SAC, APAP, and DFX did not significantly affect the fluorescent signals, which is consistent with the fact that these chemicals are considered to not be neurotoxicants. However, we were not able to detect the significant change of these fluorescent signals in zebrafish exposed to CPM, $\mathrm{RA}, \mathrm{MeHg}$, and DEX that have been reported to be developmental neurotoxicants [42,43]. Hierarchical clustering of the fluorescence parameters for each chemical (Figure 5) revealed tight clustering of (i) TSA, VPA, and CBZ; (ii) CPM, NCT, and CPF; (iii) BPA and RA; and (iv) DEX, SAC, APAP, and DFX; reflecting on the trend of (i) increase of RFP and decrease of YFP and CFP; (ii) decrease of YFP and CFP; (iii) decrease of RFP and CFP; and (iv) little change of CFP, RFP, and YFP, respectively. $\mathrm{MeHg}$, 
whose average signal of YFP was relatively high, was not tightly clustered with other chemicals. Thus, the triple-Tg zebrafish line appears to be capable of discriminating between developmental neurotoxicants that affect different cell types within the CNS.

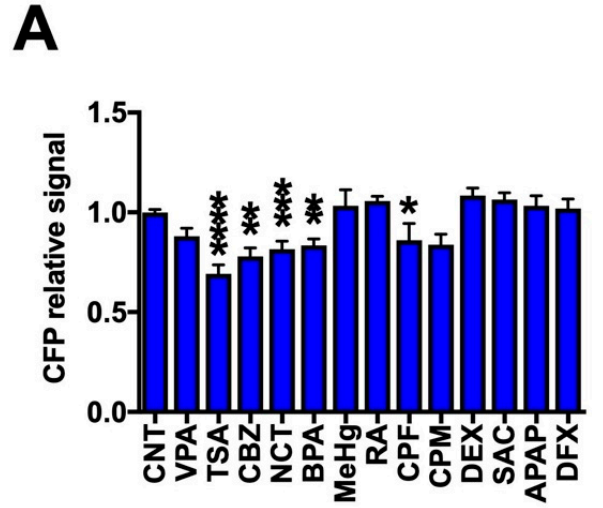

B

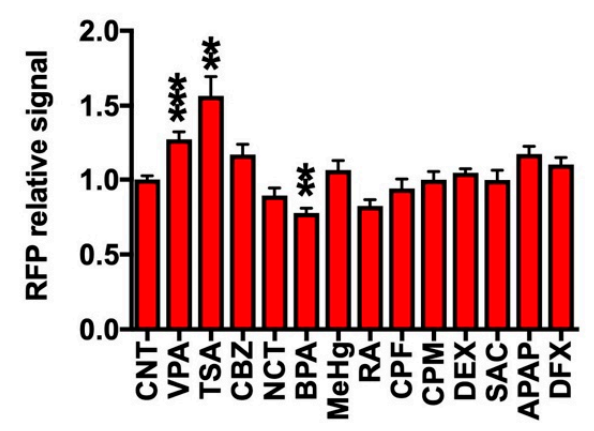

C

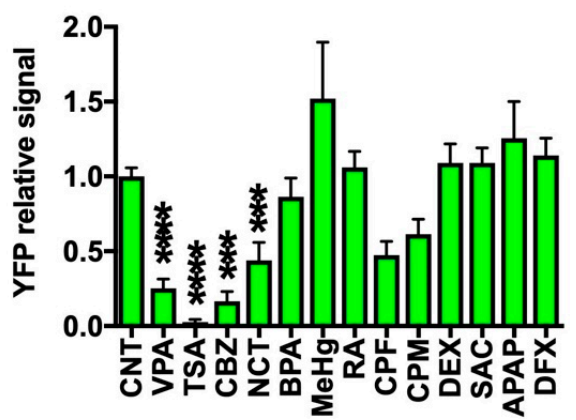

D

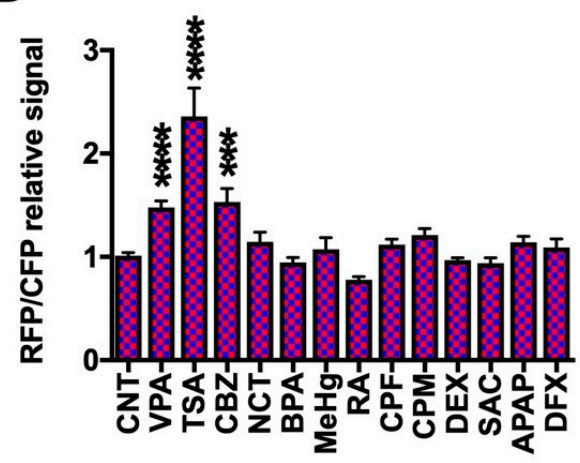

$\mathbf{E}$

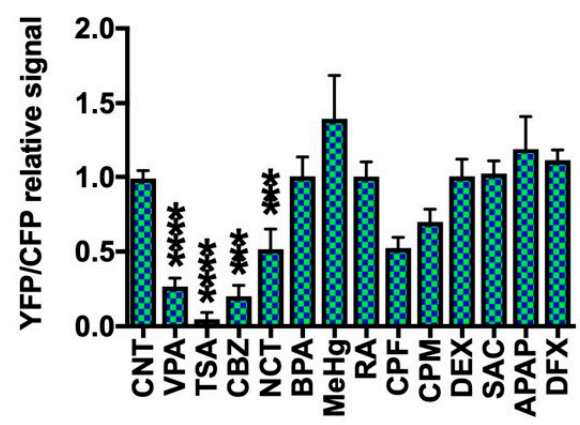

$\mathbf{F}$

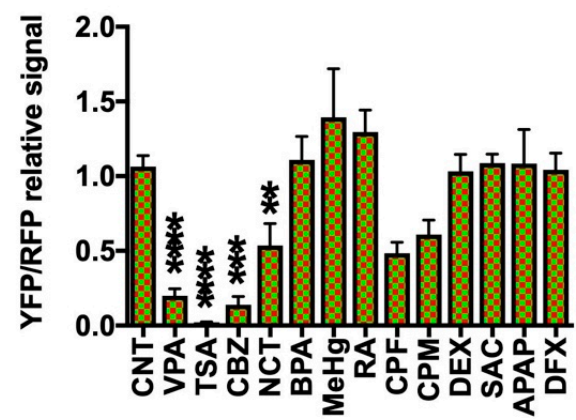

Figure 4. Quantification of in vivo fluorescence imaging of triple-Tg zebrafish exposed to chemicals at the maximum tolerable concentrations during early development. Triple-Tg zebrafish were treated as described for Figure 3 and subjected to in vivo fluorescence imaging at $5 \mathrm{dpf}$. The fluorescence signals for CFP (A), RFP (B), YFP (C), RFP/CFP ratio (D), YFP/CFP ratio (E), and YFP/RFP ratio (F) were quantified and normalized to the mean signals in the untreated control zebrafish group (CNT). ${ }^{*} p<0.05,{ }^{* *} p<0.01,{ }^{* * *} p<0.001,{ }^{* * * *} p<0.0001$. Data are presented as the mean \pm SEM of $4-77$ zebrafish/chemical. 


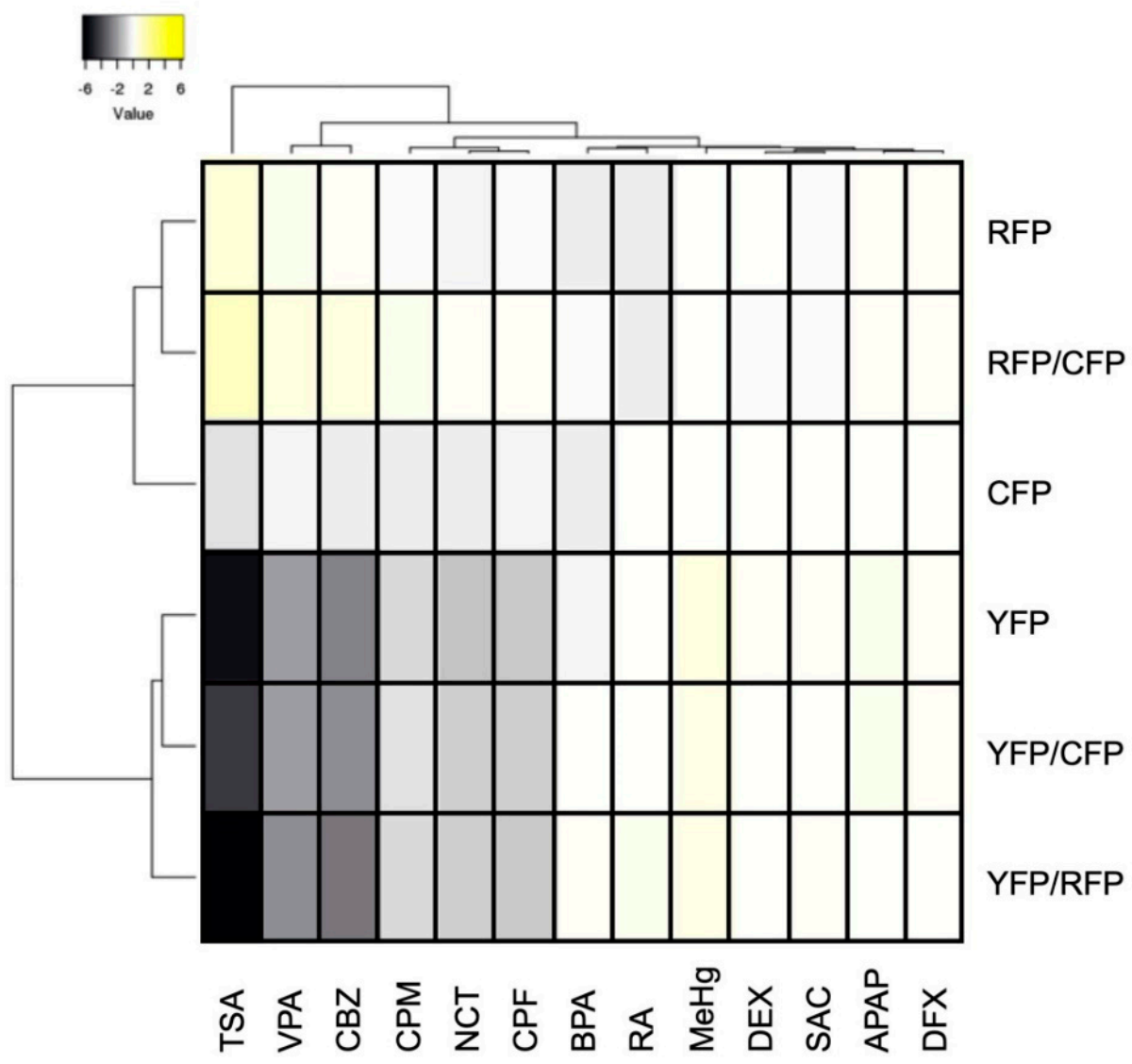

Figure 5. Hierarchical clustering of chemicals based on their effects on Cerulean (CFP), mCherry (RFP), and mCitrine (YFP) expression in triple-Tg zebrafish. The normalized score of six fluorescence parameters (CFP, RFP, YFP, RFP/CFP, YFP/CFP, and YFP/RFP) from triple-Tg zebrafish exposed to chemicals at their MTC from $12 \mathrm{hpf}$ to $5 \mathrm{dpf}$ were subjected to hierarchical clustering using Manhattan distance with average linkage.

\section{Discussion}

Here, we report the development of a triple-Tg zebrafish line expressing Cerulean, mCherry, and mCitrine in neurons, astrocytes, and oligodendrocytes, respectively, and we demonstrate with in vivo fluorescence imaging that the line can be used to assess the effects of chemicals on the differentiation of neural progenitors into the three cell types. Thus, this triple-Tg zebrafish line may be a useful tool for DNT testing during neuronal differentiation.

Previous work has demonstrated the utility of Tg zebrafish lines expressing a single fluorescent protein in neurons for DNT testing. For example, animals expressing green fluorescent protein (GFP) under the control of the neuron-specific hb9 promoter were successfully used to demonstrate a dose-dependent reduction of axon length following exposure to ethanol [57], which is consistent with the DNT of ethanol in humans [58,59]. Similarly, a zebrafish line with nkx2.2a-driven expression of GFP in motor neurons was used to demonstrate significant shortening of axons following exposure to five neurotoxicants at concentrations that did not cause external malformations [60]. Another Tg zebrafish line expressing mCitrine in oligodendrocytes under the control of the mbp promoter was able to demonstrate not only the DNT of antithyroid drugs (methimazole and propylthiouracil), but also the stimulatory effects of thyroid hormone on oligodendrocyte differentiation [61]. A zebrafish line with HuC/elavl3-driven expression of GFP in neurons and olig2-driven dsRed expression in oligodendrocytes was successfully used to detect DNT of trimethyltin chloride [62]. To our knowledge, however, there have been no reports of DNT testing using a triple-Tg zebrafish line expressing distinct fluorescent proteins in neurons, astrocytes, and oligodendrocytes. 
Here, we selected 13 chemicals recommended as reference compounds to validate newly developed assays for DNT [42,43]; of which 10 have been reported to be neurotoxicants and 3 have no reported neurotoxicity. By quantifying the relative fluorescence of each reporter protein in chemical-exposed zebrafish, we were able to detect significant effects of 6 of the 10 putative neurotoxicants (VPA, TSA, CBZ, NCT, CPF, and BPA) on neuronal differentiation.

Based on their fluorescent signatures, VPA, TSA, and CBZ appeared to inhibit and stimulate the differentiation of oligodendrocytes and astrocytes, respectively. These results are consistent with previous studies in rodents demonstrating that VPA inhibits and stimulates neural progenitor cell differentiation to oligodendrocytes and astrocytes, respectively [44,45]. Activation of histone deacetylase 3 (HDAC3) has been reported to stimulate and inhibit the differentiation of rat neural progenitors to oligodendrocytes and astrocytes, respectively [63]. Interestingly, VPA, TSA, and CBZ are known to inhibit HDAC3 [64-66], suggesting that they may affect oligodendrocyte and astrocyte differentiation by suppressing HDAC 3 activity in neural progenitor cells. Our results are also consistent with the report demonstrating the inhibition of neurogenesis by TSA and other HDAC inhibitors [67].

We found that exposure of the triple-Tg zebrafish to NCT, a nicotinic acetylcholine receptor (nAChR) agonist, suppressed the differentiation of oligodendrocytes, as indicated by the fluorescent signatures. This finding is consistent with previous work showing that gestational exposure of rats to NCT resulted in significantly reduced expression of myelin genes, including Mbp, in the prefrontal cortex and nucleus accumbens of juvenile rats [46]. We also found that CPF, an acetylcholinesterase (AChE) inhibitor, showed a trend to suppress the differentiation of oligodendrocytes in the triple-Tg zebrafish. In a similar study, exposure of rats to the AChE inhibitor carbofuran from gestational day 7 onwards also led to impaired myelination in the hippocampus on postnatal day 21 [47]. The results of the present study are consistent with these reports, collectively suggesting that misregulation of nAChR function during development may be associated with impaired oligodendrocyte differentiation. Our results are also consistent with the report demonstrating the inhibition of neurogenesis by NCT and CPF $[68,69]$. Hierarchical clustering of the fluorescence parameters revealed that CPM was tightly clustering with NCT and CPF. It is noteworthy that CPM inhibits PAX6 and hedgehog signaling, key players in the differentiation into neurons and oligodendrocytes, respectively $[48,70,71]$.

Triple-Tg zebrafish exposed to BPA showed significantly decreased Cerulean and mCherry expression, indicative of reduced the differentiation into neurons and astrocytes, respectively. BPA attenuate the expression of Pax6 [49], suggesting that BPA may decrease the differentiation into neurons by disrupting Pax6 functions. The mechanisms of how BPA decrease the differentiation into astrocytes remain to be elucidated. RFP signal seems to be reduced by RA in the hierarchical clustering. This is consistent with a previous report demonstrating that RA suppressed the expression of GFAP in embryonic day 13 cortical progenitor cells [55], although the reduction was not statistically significant in this study.

We were not able to detect significant effects of MeHg and DEX on the neuronal differentiation in the triple- $\mathrm{Tg}$ zebrafish. It has been demonstrated that $\mathrm{MeHg}, \mathrm{DEX}$, and RA significantly affect neural progenitor differentiation in rodents [55,56,72]. In this study, zebrafish were exposed to these chemicals from $12 \mathrm{hpf}$ to $5 \mathrm{dpf}$. We can change the exposure time and concentration of chemicals. Further studies are needed to optimize the assessment of DNT using triple-Tg zebrafish. The optimization could also stimulate using the triple-Tg zebrafish for drug discovery targeting neural regeneration and other studies focusing on neuronal differentiation.

Finally, developmental exposure of triple-Tg zebrafish to SAC, APAP, and DFX had no significant effects on fluorescent protein expression, which is consistent with previous demonstrations that these chemicals are not developmental neurotoxicants [42,43].

This study has several limitations. First, we used eno2, gfap, and mbp as markers for neurons, astrocytes, and oligodendrocytes, respectively. Although these genes are well-established neuronal cell type-specific markers in mammals [73-75] and zebrafish [36-38], they are expressed at relatively late stages of neuronal differentiation [73-75]. Therefore, the triple-Tg zebrafish developed in this 
study may not be suitable for DNT testing in the early stages of neuronal differentiation. Second, zebrafish can absorb most, but not all, chemicals from the surrounding medium, and chemicals with low lipophilicity (high hydrophilicity) tend to be poorly absorbed $[4,33,76]$. Third, the molecular mechanisms underlying the DNT of each chemical on neuronal differentiation in the Tg zebrafish remain to be clarified. Genome editing technology is easily performed in zebrafish, making it feasible to generate gene-specific knockout animals. Integrative analysis of various knockout and transgenic zebrafish lines will make it possible to clarify the adverse outcome pathways underlying the effects of neurotoxicants on neuronal differentiation.

In summary, we have generated a triple-Tg zebrafish line to enable DNT screening of neuron, astrocyte, and oligodendrocyte differentiation within 1 week. Further studies with an expanded chemical library will be required to rigorously assess the utility of in vivo fluorescent imaging of triple-Tg zebrafish as an alternative method to assess DNT.

\section{Materials and Methods}

\subsection{Ethics Statement}

Mie University Institutional Animal Care and Use Committee guidelines state that no approval is required for experiments using zebrafish. Nonetheless, the animal experiments described in this manuscript conform to the ethical guidelines established by the committee.

\subsection{Compounds}

Valproic acid (VPA), deferoxamine (DFX), acetaminophen (APAP), nicotine (NCT), dexamethasone (DEX), chlorpyrifos (CPF), and methyl mercury (MeHg) were purchased from Sigma (St. Louis, MO, USA). Cyclopamine (CPM) and retinoic acid (RA) were purchased from Wako (Osaka, Japan). Trichostatin A (TSA), carbamazepine (CBZ), bisphenol A (BPA), and saccharin (SAC) were purchased from Tokyo Kasei (Tokyo, Japan). With the exception of CPM, DFX, and APAP, stock solutions were prepared in dimethyl sulfoxide (DMSO; Nacalai, Kyoto, Japan). CPM was dissolved in ethanol (Wako), and DFX and APA were dissolved in in $0.3 \times$ Danieau's solution $(19.3 \mathrm{mM} \mathrm{NaCl}, 0.23 \mathrm{mM} \mathrm{KCl}, 0.13 \mathrm{mM}$ $\mathrm{MgSO}_{4}, 0.2 \mathrm{mM} \mathrm{Ca}\left(\mathrm{NO}_{3}\right)_{2}, 1.7 \mathrm{mM}$ HEPES, $\mathrm{pH}$ 7.2). For experiments, chemicals were serially diluted in $0.3 \times$ Danieau's solution. Controls contained the same final concentrations of vehicle.

\subsection{Zebrafish Husbandry}

Zebrafish were maintained according to standard methods as described previously [61,77]. Briefly, zebrafish were raised at $28.5 \pm 0.5^{\circ} \mathrm{C}$ with a $14 / 10 \mathrm{~h}$ light/dark cycle. Embryos were obtained by natural mating and cultured in $0.3 \times$ Danieau's solution.

\subsection{Generation of $\operatorname{Tg}$ (eno2:Cerulean, gfap:mCherry, mbp:mCitrine) Zebrafish}

We used an albino zebrafish line [78] (Max Planck Institute for Developmental Biology, Tübingen, Germany) to generate the Tg zebrafish line. The coding regions of Cerulean, mCherry, and mCitrine were amplified by PCR from pCS2+8NCerulean, pCS2+8NmCherry, and pCS2+8NmCitrine plasmids, respectively (Addgene, Cambridge, MA, USA), and cloned into a Tol2 vector using the In-fusion HD cloning kit (Takara Bio, Shiga, Japan) to generate three circular plasmids (pT2-Cerulean, pT2-mCherry, and pT2-mCitrine). Briefly, a fragment (bp 3017 to 1088) of pT2AL200R150G [40] was amplified by inverse PCR and fused with the coding regions of Cerulean, mCherry, or mCitrine to generate the circular plasmids. The promoters of zebrafish eno2 ( -3783 to $-3723 \mathrm{bp}$ ) [36] and mbp ( -1873 to $80 \mathrm{bp}$ ) [38] were synthesized (Invitrogen, Carlsbad, CA, USA) and cloned into the relevant pT2 plasmid using the In-fusion HD cloning kit (Takara Bio, Shiga, Japan). The promoter of zebrafish gfap [37] was amplified from pEGFP-gfap (Intron1/5'/Exon1-zebrafish; Addgene) by PCR and cloned into pT2-mCherry using the In-fusion HD cloning kit (Takara Bio). To generate single-Tg zebrafish, one of the three plasmids and transposase mRNA were injected into zebrafish embryos at the 1-4 
cell stage, and larvae expressing the fluorescent protein in the CNS were selected and maintained. Mature F0 single-Tg zebrafish were mated with albino zebrafish and single-Tg F1 animals were selected and maintained. Mature F1 male single-Tg zebrafish were mated with mature F1 female single-Tg zebrafish expressing a different fluorescent protein to generate F2 double-Tg zebrafish. Mature F2 double-Tg male and female zebrafish (e.g., males expressing Cerulean and mCherry and females expressing mCherry and mCitrine) were mated to generate F3 zebrafish. Finally, the F3 zebrafish with eno2-driven Cerulean expression in neurons, gfap-driven mCherry expression in astrocytes, and mbp-driven mCitrine expression in oligodendrocytes were selected by in vivo fluorescence imaging at $5 \mathrm{dpf}$, maintained, and analyzed.

\subsection{Exposure of Triple-Tg Zebrafish to Chemicals}

Mature triple-Tg zebrafish were mated with the albino mutant line and the resulting embryos (40 per well in 6-well plates) were exposed to serial dilutions of the chemicals from $12 \mathrm{hpf}$ to $5 \mathrm{dpf}$ without changing the medium. zebrafish were then imaged using a nikon smz800 stereoscopic microscope according to a fish embryo acute toxicity test guideline (oecd tg236) [41]. the highest chemical concentration that did not induce lethality (noec) was taken as the maximum tolerable concentration $(\mathrm{mtc})$ for assessment of dnt.

\subsection{In Vivo Imaging of Triple-Tg Zebrafish}

Mature triple-Tg zebrafish were mated with the albino mutant line, and the embryos (40/well in 6-well plates) were exposed to chemicals at the MTCs from $12 \mathrm{hpf}$ to $5 \mathrm{dpf}$ without changing the medium. At $5 \mathrm{dpf}$, the larvae were transferred to fresh $0.3 \times$ Danieau's solution containing 2-phenoxyethanol (500 ppm) to be anesthetized and then transferred to glass slides. A few drops of $3 \%$ low-melting agarose solution were placed over the larvae, and the animals were immediately oriented with the dorsal side up. The zebrafish were then observed using an epifluorescence microscope (SMZ25, Nikon, Tokyo, Japan) with the following filters: CFP (Ex/Em 425-445/457-500 nm), RFP (Ex/Em 530-560/590-650 nm), and YFP (Ex/Em 458-512/529-550 nm) to detect Cerulean (Ex/Em 433/475 nm), mCherry (Ex/Em 587/610 nm), and mCitrine (Ex/Em 516/529 nm) fluorescence, respectively. Fluorescent signals were quantified using Volocity (Perkin Elmer, Cambridge, MA, USA). A region of interest was placed around the Cerulean fluorescence observed in the brain and spinal cord in the 256 gray-scale (0-255) image. The areas of CFP, RFP, and YFP fluorescence within the region of interest that contained pixels above the intensity threshold ( 30 for Cerulean and mCherry, 20 for mCitrine) were measured. Single-protein signals and the ratios of RFP/CFP, YFP/CFP, and YFP/RFP signals were calculated.

\subsection{Statistical Analysis}

CFP, RFP, YFP, RFP/CFP, YFP/CFP, and YFP/RFP fluorescent signals in each treated zebrafish were normalized to the average signals in the untreated control group. Data are shown as the mean \pm standard error of the mean (SEM). We performed D'Agostino-Pearson normality test to examine the distribution of these data related to each chemical. The test revealed that these data were not always normally distributed. We, therefore, used Kruskal-Wallis test and Dunnett's multiple comparisons test to examine the differences between group means. Hierarchical clustering of log-transformed normalized fluorescent signals was performed using Heatmapper [79] with Manhattan as the distance measurement method and average linkage as the clustering method. Statistical analyses were performed using Prism 7 software (GraphPad, La Jolla, CA, USA).

Supplementary Materials: The following are available online at http://www.mdpi.com/1424-8247/12/4/145/s1, Table S1: Summary of in vivo fluorescence imaging of triple-Tg zebrafish.

Author Contributions: Conceptualization, Y.N.; Methodology, Y.N.; Validation, J.K., T.S., Y.A., M.I., and Y.N.; Formal Analysis, Y.N.; Investigation, J.K., T.S., Y.A., M.I., and Y.N.; Resources, K.N., T.T., and Y.N.; Data Curation, Y.N.; Writing-Original Draft Preparation, Y.N.; Visualization, Y.N.; Supervision, Y.N.; Project Administration, Y.N.; Funding Acquisition, T.S. and Y.N. 
Funding: This work was supported in part by the Long-range Research Initiative of the Japan Chemical Industrial Association (13_PT01-01), the Japan Society for the Promotion of Science KAKENHI (19K07318, 18K06890), and Takeda Science Foundation.

Acknowledgments: We are grateful to Hirokazu Takahashi (Lion Corporation), Tatsuhiro Niino (LSI Medience Corporation), and Shunji Nakai (Sumitomo Chemical) for excellent advice on the assessment of developmental neurotoxicity. We would like to thank Rie Ikeyama for secretarial assistance and Anne M. O'Rourke, from Edanz Group (www.edanzediting.com/ac) for editing a draft of this manuscript.

Conflicts of Interest: The authors declare no conflicts of interest.

\section{References}

1. Costa, L.G.; Steardo, L.; Cuomo, V. Structural Effects and Neurofunctional Sequelae of Developmental Exposure to Psychotherapeutic Drugs: Experimental and Clinical Aspects. Pharmacol. Rev. 2004, 56, 103-147. [CrossRef] [PubMed]

2. Bal-Price, A.; Hogberg, H.T.; Crofton, K.M.; Daneshian, M.; FitzGerald, R.E.; Fritsche, E.; Heinonen, T.; Hougaard Bennekou, S.; Klima, S.; Piersma, A.H.; et al. Prenatal exposure to drugs: Effects on brain development and implications for policy and education. Nat. Rev. Neurosci. 2009, 10, 303.

3. Crofton, K.M.; Mundy, W.R.; Shafer, T.J. Developmental neurotoxicity testing: A path forward. Congenit. Anom. 2012, 52, 140-146. [CrossRef] [PubMed]

4. Nishimura, Y.; Murakami, S.; Ashikawa, Y.; Sasagawa, S.; Umemoto, N.; Shimada, Y.; Tanaka, T. Zebrafish as a systems toxicology model for developmental neurotoxicity testing. Congenit. Anom. 2015, 55, 1-16. [CrossRef] [PubMed]

5. Aoyama, H.; Takahashi, N.; Shutoh, Y.; Motomura, A.; Crofton, K.M. Developmental Neurotoxicology: History and Outline of Developmental Neurotoxicity Study Guidelines. Food Saf. 2015, 3, 48-61. [CrossRef]

6. Grandjean, P.; Abdennebi-Najar, L.; Barouki, R.; Cranor, C.F.; Etzel, R.A.; Gee, D.; Heindel, J.J.; Hougaard, K.S.; Hunt, P.; Nawrot, T.S.; et al. Timescales of developmental toxicity impacting on research and needs for intervention. Basic Clin. Pharmacol. Toxicol. 2018, 125, 70-80. [CrossRef] [PubMed]

7. Tohyama, C. Developmental neurotoxicity test guidelines: Problems and perspectives. J. Toxicol. Sci. 2016, 41, SP69-SP79. [CrossRef] [PubMed]

8. Costa, L.G.; Chang, Y.C.; Cole, T.B. Developmental Neurotoxicity of Traffic-Related Air Pollution: Focus on Autism. Curr. Environ. Health Rep. 2017, 4, 156-165. [CrossRef]

9. Allen, J.L.; Oberdörster, G.; Morris-Schaffer, K.; Wong, C.; Klocke, C.; Sobolewski, M.; Conrad, K.; Mayer-Proschel, M.; Cory-Slechta, D.A. Developmental neurotoxicity of inhaled ambient ultrafine particle air pollution: Parallels with neuropathological and behavioral features of autism and other neurodevelopmental disorders. NeuroToxicology 2017, 59, 140-154. [CrossRef]

10. Tamm, C.; Ceccatelli, S. Mechanistic insight into neurotoxicity induced by developmental insults. Biochem. Biophys. Res. Commun. 2017, 482, 408-418. [CrossRef]

11. Myhre, O.; Låg, M.; Villanger, G.D.; Oftedal, B.; Øvrevik, J.; Holme, J.A.; Aase, H.; Paulsen, R.E.; Bal-Price, A.; Dirven, H. Early life exposure to air pollution particulate matter (PM) as risk factor for attention deficit/hyperactivity disorder (ADHD): Need for novel strategies for mechanisms and causalities. Toxicol. Appl. Pharmacol. 2018, 354, 196-214. [CrossRef] [PubMed]

12. Bal-Price, A.; Pistollato, F.; Sachana, M.; Bopp, S.K.; Munn, S.; Worth, A. Strategies to improve the regulatory assessment of developmental neurotoxicity (DNT) using in vitro methods. Toxicol. Appl. Pharmacol. 2018, 354, 7-18. [CrossRef] [PubMed]

13. Modabbernia, A.; Velthorst, E.; Reichenberg, A. Environmental risk factors for autism: An evidence-based review of systematic reviews and meta-analyses. Mol. Autism 2017, 8, 13. [CrossRef] [PubMed]

14. Heyer, D.B.; Meredith, R.M. Environmental toxicology: Sensitive periods of development and neurodevelopmental disorders. Neurotoxicology 2017, 58, 23-41. [CrossRef] [PubMed]

15. Cattane, N.; Richetto, J.; Cattaneo, A. Prenatal exposure to environmental insults and enhanced risk of developing Schizophrenia and Autism Spectrum Disorder: Focus on biological pathways and epigenetic mechanisms. Neurosci. Biobehav. Rev. 2018. [CrossRef] [PubMed]

16. OECD. Oecd Guidelines for the Testing of Chemicals Test No. 426; Developmental Neurotoxicity Study; OECD iLibrary: Paris, France, 2007. 
17. Tsuji, R.; Crofton, K. Developmental neurotoxicity guideline study: Issues with methodology, evaluation and regulation. Congenit. Anom. 2012, 52, 122-128. [CrossRef] [PubMed]

18. Kuwagata, M. Current problems of in vivo developmental neurotoxicity tests and a new in vivo approach focusing on each step of the developing central nervous system. Congenit. Anom. 2012, 52, 129-139. [CrossRef] [PubMed]

19. Aoyama, H. Developmental neurotoxicity testing: Scientific approaches towards the next generation to protect the developing nervous system of children. An overview of the Developmental Neurotoxicity Symposium in 2011. Congenit. Anom. 2012, 52, 119-121. [CrossRef]

20. Nishimura, Y.; Inoue, A.; Sasagawa, S.; Koiwa, J.; Kawaguchi, K.; Kawase, R.; Maruyama, T.; Kim, S.; Tanaka, T. Using zebrafish in systems toxicology for developmental toxicity testing. Congenit. Anom. 2016, 56, 18-27. [CrossRef]

21. Fritsche, E.; Grandjean, P.; Crofton, K.M.; Aschner, M.; Goldberg, A.; Heinonen, T.; Hessel, E.V.; Hogberg, H.T.; Bennekou, S.H.; Lein, P.J.; et al. Consensus statement on the need for innovation, transition and implementation of developmental neurotoxicity (DNT) testing for regulatory purposes. Toxicol. Appl. Pharmacol. 2018, 354, 3-6. [CrossRef]

22. Bal-Price, A. Recommendation on test readiness criteria for new approach methods in toxicology: Exemplified for developmental neurotoxicity_suppl. ALTEX 2018, 35, 306. [CrossRef]

23. Miller, G.W.; Chandrasekaran, V.; Yaghoobi, B.; Lein, P.J. Opportunities and challenges for using the zebrafish to study neuronal connectivity as an endpoint of developmental neurotoxicity. NeuroToxicology 2018, 67, 102-111. [CrossRef] [PubMed]

24. Li, J.; Settivari, R.; LeBaron, M.J.; Marty, M.S. An industry perspective: A streamlined screening strategy using alternative models for chemical assessment of developmental neurotoxicity. NeuroToxicology 2019, 73, 17-30. [CrossRef] [PubMed]

25. Sachana, M.; Bal-Price, A.; Crofton, K.M.; Bennekou, S.H.; Shafer, T.J.; Behl, M.; Terron, A. International Regulatory and Scientific Effort for Improved Developmental Neurotoxicity Testing. Toxicol. Sci. 2019, 167, 45-57. [CrossRef] [PubMed]

26. Pamies, D.; Barreras, P.; Block, K.; Makri, G.; Kumar, A.; Wiersma, D.; Smirnova, L.; Zang, C.; Bressler, J.; Christian, K.M.; et al. A human brain microphysiological system derived from induced pluripotent stem cells to study neurological diseases and toxicity. ALTEX 2017, 34, 362-376. [CrossRef]

27. Fritsche, E.; Barenys, M.; Klose, J.; Masjosthusmann, S.; Nimtz, L.; Schmuck, M.; Wuttke, S.; Tigges, J. Current Availability of Stem Cell-Based In Vitro Methods for Developmental Neurotoxicity (DNT) Testing. Toxicol. Sci. 2018, 165, 21-30. [CrossRef]

28. Sakuratani, Y.; Horie, M.; Leinala, E. Integrated Approaches to Testing and Assessment: OECD Activities on the Development and Use of Adverse Outcome Pathways and Case Studies. Basic Clin. Pharmacol. Toxicol. 2018, 123, 20-28. [CrossRef]

29. Sandstrom, J.; Eggermann, E.; Charvet, I.; Roux, A.; Toni, N.; Greggio, C.; Broyer, A.; Monnet-Tschudi, F.; Stoppini, L. Development and characterization of a human embryonic stem cell-derived 3D neural tissue model for neurotoxicity testing. Toxicol. In Vitro 2017, 38, 124-135. [CrossRef]

30. Joshi, P.; Yu, K.N.; Kang, S.Y.; Kwon, S.J.; Kwon, P.S.; Dordick, J.S.; Kothapalli, C.R.; Lee, M.Y. 3D-cultured neural stem cell microarrays on a micropillar chip for high-throughput developmental neurotoxicology. Exp. Cell Res. 2018, 370, 680-691. [CrossRef]

31. Pei, Y.; Sierra, G.; Sivapatham, R.; Swistowski, A.; Rao, M.S.; Zeng, X. A platform for rapid generation of single and multiplexed reporters in human iPSC lines. Sci. Rep. 2015, 5, 9205. [CrossRef]

32. Peterson, R.T.; Macrae, C.A. Systematic approaches to toxicology in the zebrafish. Annu. Rev. Pharmacol. Toxicol. 2012, 52, 433-453. [CrossRef] [PubMed]

33. Padilla, S.; Corum, D.; Padnos, B.; Hunter, D.; Beam, A.; Houck, K.; Sipes, N.; Kleinstreuer, N.; Knudsen, T.; Dix, D.; et al. Zebrafish developmental screening of the ToxCast ${ }^{\mathrm{TM}}$ Phase I chemical library. Reprod. Toxicol. 2012, 33, 174-187. [CrossRef] [PubMed]

34. Diekmann, H.; Hill, A. ADMETox in zebrafish. Drug Discov. Today 2013, 10, e31. [CrossRef]

35. d'Amora, M.; Giordani, S. The Utility of Zebrafish as a Model for Screening Developmental Neurotoxicity. Front. Neurosci. 2018, 12, 976. [CrossRef] [PubMed] 
36. Bai, Q.; Garver, J.A.; Hukriede, N.A.; Burton, E.A. Generation of a transgenic zebrafish model of Tauopathy using a novel promoter element derived from the zebrafish eno2 gene. Nucleic Acids Res. 2007, 35, 6501-6516. [CrossRef]

37. Bernardos, R.L.; Raymond, P.A. GFAP transgenic zebrafish. Gene Expr. Patterns 2006, 6, 1007-1013. [CrossRef]

38. Jung, S.H.; Kim, S.; Chung, A.Y.; Kim, H.T.; So, J.H.; Ryu, J.; Park, H.C.; Kim, C.H. Visualization of myelination in GFP-transgenic zebrafish. Dev. Dyn. 2010, 239, 592-597. [CrossRef]

39. Brockway, N.L.; Cook, Z.T.; O'Gallagher, M.J.; Tobias, Z.J.C.; Gedi, M.; Carey, K.M.; Unni, V.K.; Pan, Y.A.; Metz, M.R.; Weissman, T.A. Multicolor lineage tracing using in vivo time-lapse imaging reveals coordinated death of clonally related cells in the developing vertebrate brain. Dev. Biol. 2019, 453, 130-140. [CrossRef]

40. Kawakami, K. Tol2: A versatile gene transfer vector in vertebrates. Genome Biol. 2007, 8, S7. [CrossRef]

41. OECD. Oecd Guidelines for the Testing of Chemicals Test No. 236; Fish Embryo Acute Toxicity Test; OECD iLibrary: Paris, France, 2013.

42. Mundy, W.R.; Padilla, S.; Breier, J.M.; Crofton, K.M.; Gilbert, M.E.; Herr, D.W.; Jensen, K.F.; Radio, N.M.; Raffaele, K.C.; Schumacher, K.; et al. Expanding the test set: Chemicals with potential to disrupt mammalian brain development. Neurotoxicol.Teratol. 2015, 52, 25-35. [CrossRef]

43. Aschner, M.; Ceccatelli, S.; Daneshian, M.; Fritsche, E.; Hasiwa, N.; Hartung, T.; Hogberg, H.T.; Leist, M.; Li, A.; Mundi, W.R.; et al. Reference compounds for alternative test methods to indicate developmental neurotoxicity (DNT) potential of chemicals: Example lists and criteria for their selection and use. ALTEX 2016, 34, 49-74. [CrossRef] [PubMed]

44. Shen, S.; Li, J.; Casaccia-Bonnefil, P. Histone modifications affect timing of oligodendrocyte progenitor differentiation in the developing rat brain. J. Cell Biol. 2005, 169, 577-589. [CrossRef] [PubMed]

45. Asano, H.; Aonuma, M.; Sanosaka, T.; Kohyama, J.; Namihira, M.; Nakashima, K. Astrocyte Differentiation of Neural Precursor Cells is Enhanced by Retinoic Acid through a Change in Epigenetic Modification. Stem Cells 2009, 27, 2744-2752. [CrossRef] [PubMed]

46. Cao, J.; Dwyer, J.B.; Gautier, N.M.; Leslie, F.M.; Li, M.D. Central myelin gene expression during postnatal development in rats exposed to nicotine gestationally. Neurosci. Lett. 2013, 553, 115-120. [CrossRef] [PubMed]

47. Seth, B.; Yadav, A.; Tandon, A.; Shankar, J.; Chaturvedi, R.K. Carbofuran hampers oligodendrocytes development leading to impaired myelination in the hippocampus of rat brain. NeuroToxicology 2019, 70, 161-179. [CrossRef] [PubMed]

48. Halluin, C.; Madelaine, R.; Naye, F.; Peers, B.; Roussigné, M.; Blader, P. Habenular Neurogenesis in Zebrafish Is Regulated by a Hedgehog, Pax6 Proneural Gene Cascade. PLoS ONE 2016, 11, 0158210. [CrossRef] [PubMed]

49. Yang, C.W.; Chou, W.C.; Chen, K.H.; Cheng, A.L.; Mao, I.F.; Chao, H.R.; Chuang, C.Y. Visualized gene network reveals the novel target transcripts Sox2 and Pax6 of neuronal development in trans-placental exposure to bisphenol A. PLoS ONE 2014, 9, e100576. [CrossRef] [PubMed]

50. Kallur, T.; Gisler, R.; Lindvall, O.; Kokaia, Z. Pax6 promotes neurogenesis in human neural stem cells. Mol. Cell. Neurosci. 2008, 38, 616-628. [CrossRef]

51. Unoki, T.; Akiyama, M.; Kumagai, Y.; Gonçalves, F.M.; Farina, M.; Da Rocha, J.B.T.; Aschner, M. Molecular Pathways Associated With Methylmercury-Induced Nrf2 Modulation. Front. Genet. 2018, 9, 373. [CrossRef]

52. Tang, W.; Chen, X.; Liu, H.; Lv, Q.; Zou, J.; Shi, Y.; Liu, Z. Expression of Nrf2 Promotes Schwann Cell-Mediated Sciatic Nerve Recovery in Diabetic Peripheral Neuropathy. Cell. Physiol. Biochem. 2018, 46, 1879-1894. [CrossRef]

53. Annau, Z.; Cuomo, V. Mechanisms of neurotoxicity and their relationship to behavioral changes. Toxicology 1988, 49, 219-225. [CrossRef]

54. Heimfarth, L.; Delgado, J.; Mignori, M.R.; Gelain, D.P.; Moreira, J.C.F.; Pessoa-Pureur, R. Developmental neurotoxicity of the hippocampus following in utero exposure to methylmercury: Impairment in cell signaling. Arch. Toxicol. 2018, 92, 513-527. [CrossRef] [PubMed]

55. Faigle, R.; Liu, L.; Cundiff, P.; Funa, K.; Xia, Z. Opposing effects of retinoid signaling on astrogliogenesis in embryonic day 13 and 17 cortical progenitor cells. J. Neurochem. 2008, 106, 1681-1698. [CrossRef] [PubMed]

56. Slotkin, T.A.; Skavicus, S.; Seidler, F.J. Developmental neurotoxicity resulting from pharmacotherapy of preterm labor, modeled in vitro: Terbutaline and dexamethasone, separately and together. Toxicology 2018, 400, 57-64. [CrossRef] [PubMed] 
57. Kanungo, J.; Lantz, S.; Paule, M.G. In vivo imaging and quantitative analysis of changes in axon length using transgenic zebrafish embryos. Neurotoxicol. Teratol. 2011, 33, 618-623. [CrossRef] [PubMed]

58. Driscoll, C.D.; Streissguth, A.P.; Riley, E.P. Prenatal alcohol exposure: Comparability of effects in humans and animal models. Neurotoxicol. Teratol. 1990, 12, 231-237. [CrossRef]

59. Kalberg, W.O.; Provost, B.; Tollison, S.J.; Tabachnick, B.G.; Robinson, L.K.; Hoyme, H.E.; Trujillo, P.M.; Buckley, D.; Aragón, A.S.; May, P.A. Comparison of Motor Delays in Young Children With Fetal Alcohol Syndrome to Those With Prenatal Alcohol Exposure and With No Prenatal Alcohol Exposure. Alcohol. Clin. Exp. Res. 2006, 30, 2037-2045. [CrossRef] [PubMed]

60. Zhang, X.; Gong, Z. Fluorescent Transgenic Zebrafish Tg(nkx2.2a:mEGFP) Provides a Highly Sensitive Monitoring Tool for Neurotoxins. PLoS ONE 2013, 8, e55474. [CrossRef]

61. Ashikawa, Y.; Nishimura, Y.; Okabe, S.; Sasagawa, S.; Murakami, S.; Yuge, M.; Kawaguchi, K.; Kawase, R.; Tanaka, T. Activation of Sterol Regulatory Element Binding Factors by Fenofibrate and Gemfibrozil Stimulates Myelination in Zebrafish. Front. Pharmacol. 2016, 7, 206. [CrossRef]

62. Kim, J.; Kim, C.-Y.; Song, J.; Oh, H.; Kim, C.-H.; Park, J.-H. Trimethyltin chloride inhibits neuronal cell differentiation in zebrafish embryo neurodevelopment. Neurotoxicol. Teratol. 2016, 54, 29-35. [CrossRef]

63. Zhang, L.; He, X.; Liu, L.; Jiang, M.; Zhao, C.; Wang, H.; He, D.; Zheng, T.; Zhou, X.; Hassan, A.; et al. Hdac3 Interaction with p300 Histone Acetyltransferase Regulates the Oligodendrocyte and Astrocyte Lineage Fate Switch. Dev. Cell 2016, 36, 316-330. [CrossRef] [PubMed]

64. Chen, S.; Ye, J.; Chen, X.; Shi, J.; Wu, W.; Lin, W.; Lin, W.; Li, Y.; Fu, H.; Li, S. Valproic acid attenuates traumatic spinal cord injury-induced inflammation via STAT1 and NF-kappaB pathway dependent of HDAC3. J. Neuroinflamm. 2018, 15, 150. [CrossRef] [PubMed]

65. Dhoke, N.R.; Kalabathula, E.; Kaushik, K.; Geesala, R.; Sravani, B.; Das, A. Histone deacetylases differentially regulate the proliferative phenotype of mouse bone marrow stromal and hematopoietic stem/progenitor cells. Stem Cell Res. 2016, 17, 170-180. [CrossRef] [PubMed]

66. Beutler, A.S.; Li, S.; Nicol, R.; Walsh, M.J. Carbamazepine is an inhibitor of histone deacetylases. Life Sci. 2005, 76, 3107-3115. [CrossRef] [PubMed]

67. Shakèd, M.; Weissmüller, K.; Svoboda, H.; Hortschansky, P.; Nishino, N.; Wölfl, S.; Tucker, K.L. Histone Deacetylases Control Neurogenesis in Embryonic Brain by Inhibition of BMP2/4 Signaling. PLoS ONE 2008, 3, e2668. [CrossRef] [PubMed]

68. Chatterton, Z.; Hartley, B.J.; Seok, M.H.; Mendelev, N.; Chen, S.; Milekic, M.; Rosoklija, G.; Stankov, A.; Trencevsja-Ivanovska, I.; Brennand, K.; et al. In utero exposure to maternal smoking is associated with DNA methylation alterations and reduced neuronal content in the developing fetal brain. Epigenetics Chromatin 2017, 10, 4. [CrossRef] [PubMed]

69. Visan, A.; Hayess, K.; Sittner, D.; Pohl, E.E.; Riebeling, C.; Slawik, B.; Gulich, K.; Oelgeschläger, M.; Luch, A.; Seiler, A.E. Neural differentiation of mouse embryonic stem cells as a tool to assess developmental neurotoxicity in vitro. NeuroToxicology 2012, 33, 1135-1146. [CrossRef] [PubMed]

70. Incardona, J.P.; Gaffield, W.; Kapur, R.P.; Roelink, H. The teratogenic Veratrum alkaloid cyclopamine inhibits sonic hedgehog signal transduction. Development 1998, 125, 3553-3562.

71. Traiffort, E.; Zakaria, M.; Laouarem, Y.; Ferent, J. Hedgehog: A Key Signaling in the Development of the Oligodendrocyte Lineage. J. Dev. Biol. 2016, 4, 28. [CrossRef]

72. Tamm, C.; Duckworth, J.; Hermanson, O.; Ceccatelli, S. High susceptibility of neural stem cells to methylmercury toxicity: Effects on cell survival and neuronal differentiation. J. Neurochem. 2006, 97, 69-78. [CrossRef]

73. Marangos, P.J.; Schmechel, D.E.; Parma, A.M.; Goodwin, F.K. Developmental profile of neuron-specific (NSE) and non-neuronal (NNE) enolase. Brain Res. 1980, 190, 185-193. [CrossRef]

74. Avola, R.; Bramanti, V.; Tomassoni, D.; Avitabile, M.; Amenta, F. Biomarkers of glial cell proliferation and differentiation in culture. Front. Biosci. 2010, 2, 558-570. [CrossRef]

75. Jakovcevski, I.; Filipovic, R.; Mo, Z.; Rakic, S.; Zecevic, N. Oligodendrocyte Development and the Onset of Myelination in the Human Fetal Brain. Front. Neuroanat. 2009, 3, 5. [CrossRef] [PubMed]

76. Gustafson, A.L.; Stedman, D.B.; Ball, J.; Hillegass, J.M.; Flood, A.; Zhang, C.X.; Panzica-Kelly, J.; Cao, J.; Coburn, A.; Enright, B.P.; et al. Inter-laboratory assessment of a harmonized zebrafish developmental toxicology assay_Progress report on phase I. Reprod. Toxicol. 2012, 33, 155-164. [CrossRef] [PubMed] 
77. Sasagawa, S.; Nishimura, Y.; Hayakawa, Y.; Murakami, S.; Ashikawa, Y.; Yuge, M.; Okabe, S.; Kawaguchi, K.; Kawase, R.; Tanaka, T. E2F4 promotes neuronal regeneration and functional recovery after spinal cord injury in zebrafish. Front. Pharmacol. 2016, 7, 119. [CrossRef]

78. Kelsh, R.N.; Brand, M.; Jiang, Y.J.; Heisenberg, C.P.; Lin, S.; Haffter, P.; Odenthal, J.; Mullins, M.C.; Van Eeden, F.J.; Furutani-Seiki, M.; et al. Zebrafish pigmentation mutations and the processes of neural crest development. Development 1996, 123, 369-389.

79. Babicki, S.; Arndt, D.; Marcu, A.; Liang, Y.; Grant, J.R.; Maciejewski, A.; Wishart, D.S. Heatmapper: Web-enabled heat mapping for all. Nucleic Acids Res. 2016, 44, W147-W153. [CrossRef]

(C) 2019 by the authors. Licensee MDPI, Basel, Switzerland. This article is an open access article distributed under the terms and conditions of the Creative Commons Attribution (CC BY) license (http://creativecommons.org/licenses/by/4.0/). 